\title{
Static load distribution in ball bearings including the effects of temperature and fit
}

\author{
M. Ricci \\ Space Mechanics and Control Division, INPE, Brazil
}

\begin{abstract}
A numerical procedure for internal loading distribution computation in statically loaded, single-row, angular-contact ball bearings, subjected to a known combined radial and thrust load, which must be applied so that to avoid tilting between inner and outer rings, is used to find the load distribution differences between a loaded unfitted bearing at room temperature, and the same loaded bearing with interference fits, such might experience radial temperature gradients between inner and outer rings. For each step of the procedure it is required the iterative solution of $Z+2$ simultaneous non-linear equations - where $Z$ is the number of the balls - to yield exact solution for axial and radial deflections, and contact angles.
\end{abstract}

Keywords: ball, bearing, static, load, numerical, method, temperature, fit.

\section{Introduction}

Ball and roller bearings, generically called rolling bearings, are commonly used machine elements. They are employed to permit rotary motions of, or about, shafts in simple commercial devices such as bicycles, roller skates, and electric motors. They are also used in complex engineering mechanisms such as aircraft gas turbines, rolling mils, dental drills, gyroscopes, and power transmissions.

This work is devoted to study of the internal loading distribution in statically loaded single-row angular-contact ball bearings. Several researchers have studied the subject as, for example, Stribeck [1], Sjoväll [2], Jones [3] and Rumbarger [4]. The methods developed by them to calculate distribution of load among the balls and rollers of rolling bearings can be used in most bearing applications because rotational speeds are usually slow to moderate. Under these speed conditions, the effects of rolling element centrifugal forces and gyroscopic 
moments are negligible. At high speeds of rotation these body forces become significant, tending to alter contact angles and clearance. Thus, they can affect the static load distribution to a great extension.

Harris [5] described methods for internal loading distribution in statically loaded bearings addressing pure radial; pure thrust (centric and eccentric loads); combined radial and thrust load, which uses radial and thrust integrals introduced by Sjoväll; and for ball bearings under combined radial, thrust, and moment load, initially due to Jones.

There are many works describing the parameters variation models under static loads but few demonstrate such variations in practice, even under simple static loadings. The author believes that the lack of practical examples is mainly due to the inherent difficulties of the numerical procedures that, in general, deal with the resolution of several non-linear algebraic equations that must to be solved simultaneously.

In an attempt to cover this gap studies are being developed in parallel [6-10]. In this work a numerical procedure, described in [7], for internal load distribution computation in statically loaded, single-row, angular-contact ball bearings subjected to a known external combined radial and thrust load, so that no tilt is allowed between inner and outer rings, is used to find the load distribution differences between a loaded bearing with clearance fits at room temperature, and the same loaded bearing with interference fits, such might experience radial temperature gradients between inner and outer rings.

In the most usual situation, angular contact bearings would first be fitted, with interference or clearance defined at room temperature, to their respective shaft and housing; then a defined axial "hard" preload would be applied and subsequently in operation the bearings might experience radial temperature gradients between inner and outer rings.

Generally, ball bearings and other radial rolling bearings such as cylindrical roller bearings are designed to have a diametral clearance in the no-load state. Due to this radial clearance the bearing also can experience an axial play. Removal of this axial freedom causes the ball-raceway contact line to assume an oblique angle with respect to the radial plane; hence, a contact angle different from zero will occur. This angle is called free contact angle and is a function of clearance built into the unloaded bearing and the raceway groove curvatures.

Press or shrink fitting of the inner ring on the shaft causes the inner ring to expand slightly. Similarly, press fitting of the outer ring in the housing causes the former member to shrink slightly. Thus, the bearing's diametral clearance will tend to decrease.

Thermal conditions of bearing operation can also affect the diametral clearance. Heat generated by friction causes internal temperatures to rise. This in turn causes expansion of the shaft, housing, and bearing components. Depending on the shaft and housing materials and on the magnitude of thermal gradients across the bearing and these supporting structures, clearance can tend to increase or decrease. It is also apparent that the thermal environment in which a bearing operates may have a significant effect on clearance. 


\section{Static load distribution under combined radial and thrust load in ball bearings}

Having defined in other works analytical expressions for geometry of bearings and for contact stress and deformations for a given ball or roller-raceway contact (point or line loading) in terms of load, it is possible to consider how the bearing load is distributed among the rolling elements. In this section a specific load distribution consisting of a combined radial and thrust load, which must be applied to the inner ring of a statically loaded ball bearing, so that no tilt is allowed between inner and outer rings, is given.

Let a ball bearing with a number of balls, $Z$, symmetrically distributed about a pitch circle according to Fig. 1, to be subjected to a combined radial and thrust load, so that a relative axial displacement, $\delta_{a}$, and a relative radial displacement, $\delta_{r}$, between the inner and outer ring raceways may be expected. Let $\psi=0$ to be the angular position of the maximum loaded ball.

Figure 2 shows the initial and final curvature centers positions at angular position $\psi$, before and after loading, considering the centers of curvature of the raceway grooves fixed with respect to the corresponding raceway. If $\delta_{a}$ and $\delta_{r}$ are known, the contact angle at angular position $\psi$, after the combined load has been applied, is given by

$$
\beta(\psi)=\cos ^{-1}\left(\frac{A \cos \beta_{f}+\delta_{r} \cos \psi}{A+\delta_{n}}\right),
$$

where $A$ is the distance between raceway groove curvature centers for the unloaded bearing, $\beta_{f}$ is the free-contact angle and $\delta_{n}$ is the total normal deflection at the contacts.

Also, from Fig. 2,

$$
\delta_{a}=\left(A+\delta_{n}\right) \sin \beta-A \sin \beta_{f},
$$

and we can arrive in the expression for the extend of the loading zone, that is given by

$$
\psi_{l}=\cos ^{-1}\left\{\frac{A}{\delta_{r}}\left[\cos \left(\sin ^{-1}\left(\frac{\delta_{a}+A \sin \beta_{f}}{A}\right)\right)-\cos \beta_{f}\right]\right\} .
$$

From (1), the total normal approach between two raceways at angular position $\psi$, after the combined load has been applied, can be written as

$$
\delta_{n}(\psi)=A\left(\frac{\cos \beta_{f}}{\cos \beta}-1\right)+\frac{\delta_{r} \cos \psi}{\cos \beta} .
$$

From Fig. 2 and (4) it can be determined that $s$, the distance between the centers of the curvature of the inner and outer ring raceway grooves at any rolling element position $\psi$, is given by

$$
s(\psi)=A+\delta_{n}=A \frac{\cos \beta_{f}}{\cos \beta}+\frac{\delta_{r} \cos \psi}{\cos \beta} .
$$




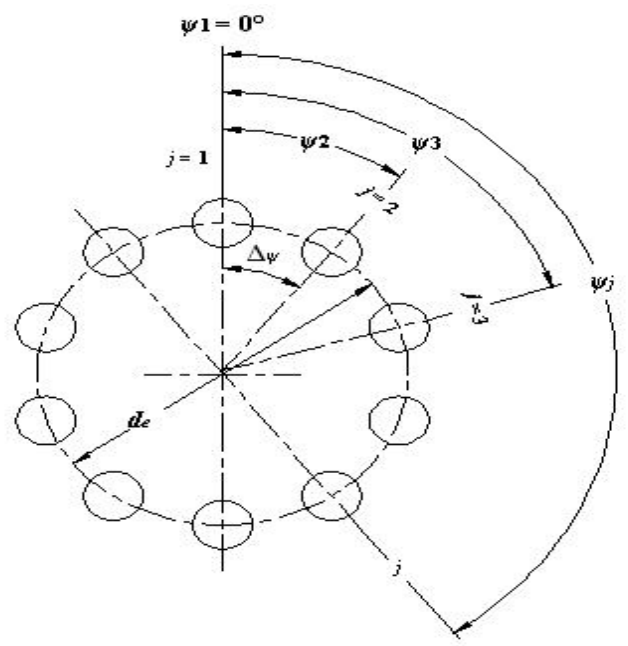

Figure 1: Ball angular positions in the radial plane that is perpendicular to the bearing's axis of rotation, $\Delta \psi=2 \pi / Z, \psi_{j}=2 \pi / Z(j-1)$.

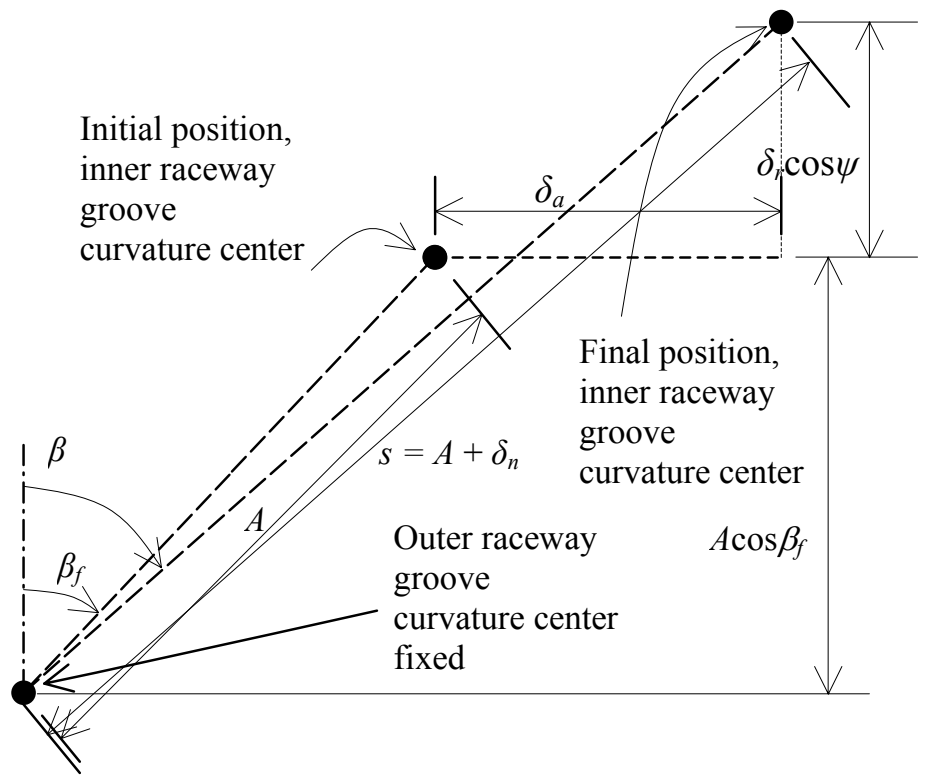

Figure 2: Initial and final curvature centers positions at angular position $\psi$, with and without applied load. 
From (2) and (5) yields, for $\psi=\psi_{j}$,

$$
\delta_{a}-\delta_{r} \tan \beta_{j} \cos \psi_{j}-A \frac{\sin \left(\beta_{j}-\beta_{f}\right)}{\cos \beta_{j}}=0, \quad j=1, \ldots, Z .
$$

From load-deflection relationship for ball bearings and (4) yields, for $\psi=\psi_{j}$,

$$
Q_{j}=K_{n j}\left[A\left(\frac{\cos \beta_{f}}{\cos \beta_{j}}-1\right)+\frac{\delta_{r} \cos \psi_{j}}{\cos \beta_{j}}\right]^{3 / 2}, j=1, \ldots, Z .
$$

If a thrust load, $F_{a}$, and a radial load, $F_{r}$, are applied then, for static equilibrium to exist

$$
\begin{gathered}
F_{a}=\sum_{j=1}^{Z} Q_{j} \sin \beta_{j}, \\
F_{r}=\sum_{j=1}^{Z} Q_{j} \cos \beta_{j} \cos \psi_{j} .
\end{gathered}
$$

Additionally, each of the normal ball load components produces a moment about of the inner ring center of mass, in the plan, which passes through the bearing rotation axis and contains the external radial load (moments about the other two perpendicular plans are self-equilibrating). For static equilibrium, the thrust load, $F_{a}$, and/or the radial load, $F_{r}$, must exert a moment, $M$, about of the inner ring center of mass, which must be equal the sum of the moments of each rolling element load, that is,

$$
M=-\sum_{j=1}^{Z} Q_{j} \sin \beta_{j}\left[\left(R_{i}+\delta_{r} \cos \psi_{j}\right) \cos \psi_{j}-\delta_{r}\right],
$$

where

$$
R_{i}=d_{e} / 2+\left(f_{i}-0.5\right) D \cos \beta_{f}
$$

expresses the locus of the centers of the inner ring raceway groove curvature radii.

Substitution of (7) into (8) yields

$$
F_{a}-\sum_{j=1}^{Z} K_{n j} \sin \beta_{j}\left(A\left(\frac{\cos \beta_{f}}{\cos \beta_{j}}-1\right)+\frac{\delta_{r} \cos \psi_{j}}{\cos \beta_{j}}\right)^{3 / 2}=0 .
$$

Similarly,

$$
F_{r}-\sum_{j=1}^{Z} K_{n j} \cos \psi_{j} \cos \beta_{j}\left(A\left(\frac{\cos \beta_{f}}{\cos \beta_{j}}-1\right)+\frac{\delta_{r} \cos \psi_{j}}{\cos \beta_{j}}\right)^{3 / 2}=0 .
$$

Equations (6), (11) and (12) are $Z+2$ simultaneous non-linear equations with unknowns $\delta_{a}, \delta_{r}$, and $\beta_{j}, j=1, \ldots, Z$. Since $K_{n j}$ are functions of final contact angle, $\beta_{j}$, the equations must be solved iteratively to yield an exact solution for $\delta_{a}, \delta_{r}$ and $\beta_{j}$. 


\section{Numerical results}

To show an application of the theory developed in this work a numerical example is presented, which uses The Newton-Rhapson method to solve the simultaneous nonlinear equations (6), (11) and (12). To take into account the effects of temperature and fitting I have considered the principal relationships between interference fittings, thermal gradients, surface finish and changes in diametral clearance, as described in [5].

I have chosen the 218 angular-contact ball bearing as example, which was also used by [5]. The 218 angular-contact ball bearing has a $0.09 \mathrm{~m}$ bore, a 0.16 $\mathrm{m}$ o.d. and is manufactured to ABEC 7 tolerance limits. The bearing is mounted on a hollow steel shaft of $0.0635 \mathrm{~m}$ bore with a $\mathrm{k} 6$ fit and in a titanium housing having a effective o.d. of $0.2032 \mathrm{~m}$ with an M6 fit. Considers that the inner ring operates at a mean temperature of $148.9^{\circ} \mathrm{C}$, that the outer ring is at $121.1^{\circ} \mathrm{C}$ and that the bearing was assembled at $21.1^{\circ} \mathrm{C}$.

There are three steps in the numerical procedure. The first, considering the bearing unfitted at assembling temperature; the second, considering the fits above at assembling temperature; and the third, considering the fits above at operational temperatures for the inner and outer rings. Before each step the geometry of the bearing is obtained from which, the nonlinear equations are solved simultaneously to obtain radial and axial deflections and contact angles.

Figs. 3 to 12 show some parameters, as functions of the applied thrust load, for the three steps of the procedure and for some values of the applied radial load.

Figs. 3 and 4 show the normal ball loads for the maximum and minimum loaded ball, respectively. There is a better loading distribution with the increase of thrust load; and there are slight decreases (increases) in normal ball load, for the maximum (minimum) loaded ball, when it passes from first to second, and

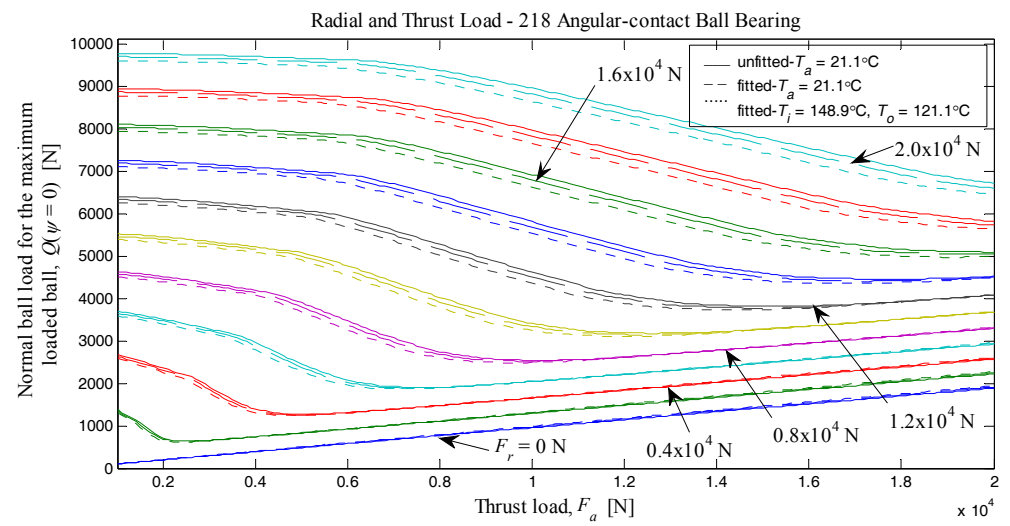

Figure 3: Normal ball load for the maximum loaded ball, $Q(\psi=0)$, as a function of the thrust load, $F_{a}$. 


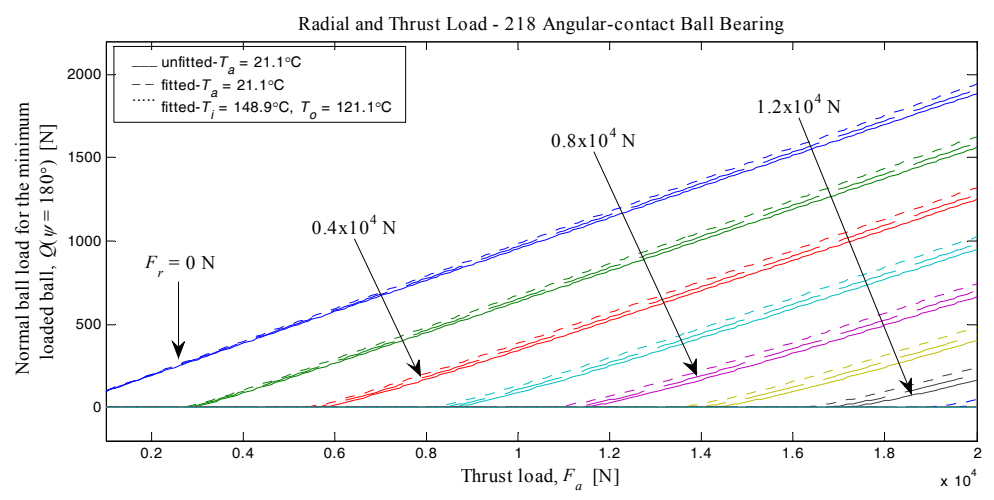

Figure 4: $\quad$ Normal ball load for the minimum loaded ball, $Q\left(\psi=180^{\circ}\right)$, as a function of the thrust load, $F_{a}$.

from second to third steps. Similar behavior is also observed for other parameters and will not be mentioned here. For this loading range, the ball at angular position $\psi=0$ is always loaded. This is not the case for the ball at angular position $\psi=180^{\circ}$. For zero applied radial load the normal ball load is the same for the maximum and minimum loaded ball.

Figs. 5 and 6 show the contact angle for the maximum and minimum loaded ball, respectively. The straight lines represent the free contact angles for the three steps of the procedure. For zero applied radial load the contact angle is always greater than the free contact angle. In this case, the bearing is under thrust loading and all balls have the same load and contact angles. If the contact angle falls below the free contact angle the balls are unloaded. With the increase in applied radial load the free contact angle ceases to be a reference for unload. In this case, the contact angle may drop to values much lower than the free contact angle value - as low as $3^{\circ}$ - for a loaded ball, or may rise to values greater than the free contact angle value, for an unloaded ball.

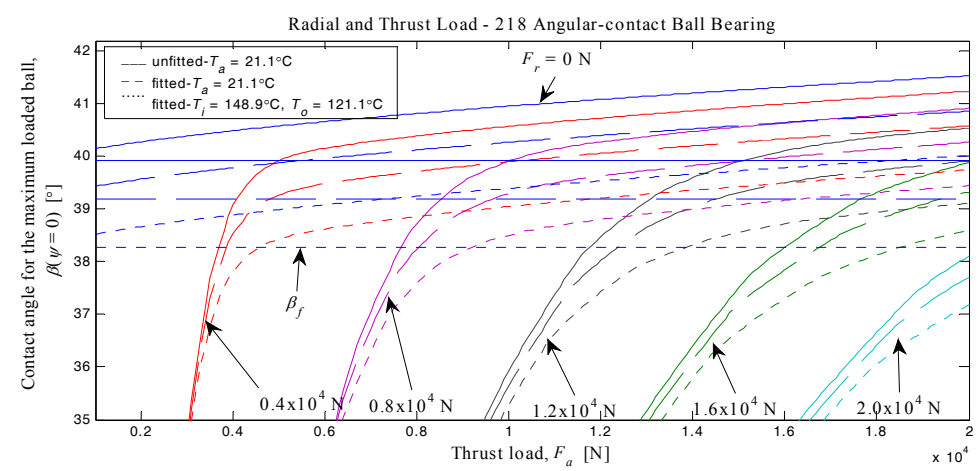

Figure 5: Contact angle for the maximum loaded ball, $\beta(\psi=0)$, as a function of the thrust load, $F_{a}$. 


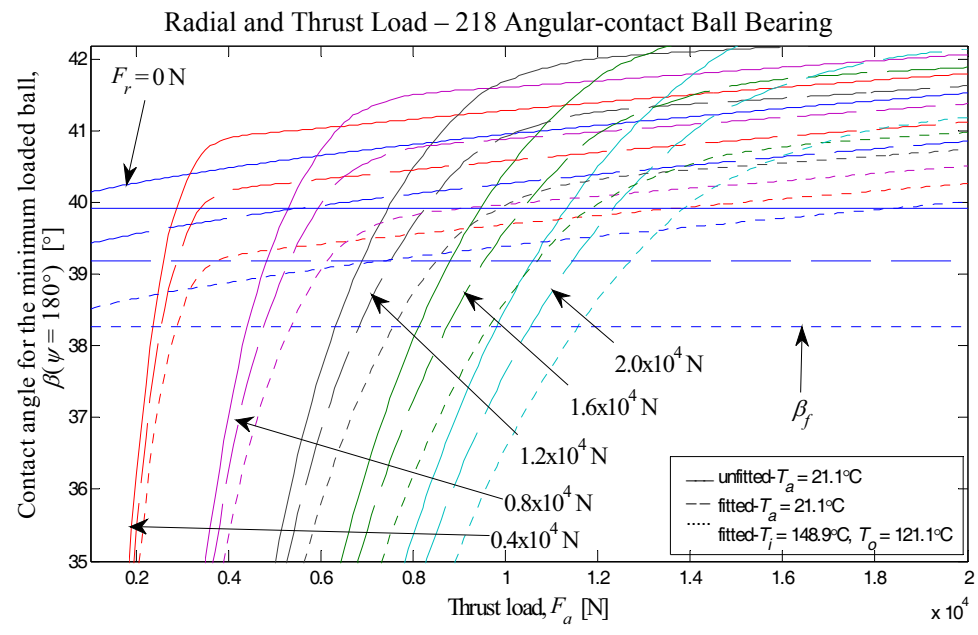

Figure 6: Contact angle for the minimum loaded ball, $\beta\left(\psi=180^{\circ}\right)$, as a function of the thrust load, $F_{a}$.

Figs. 7 and 8 show the distance between the curvature centers for the maximum and minimum loaded ball, $s$, respectively. The straight lines represent the curvature centers distance in the shifted (unload) position, $A$. Simply remove $A$ from $s$ for total deformation along the contact line. If $s$ falls below $A$ is an indication of unloading.

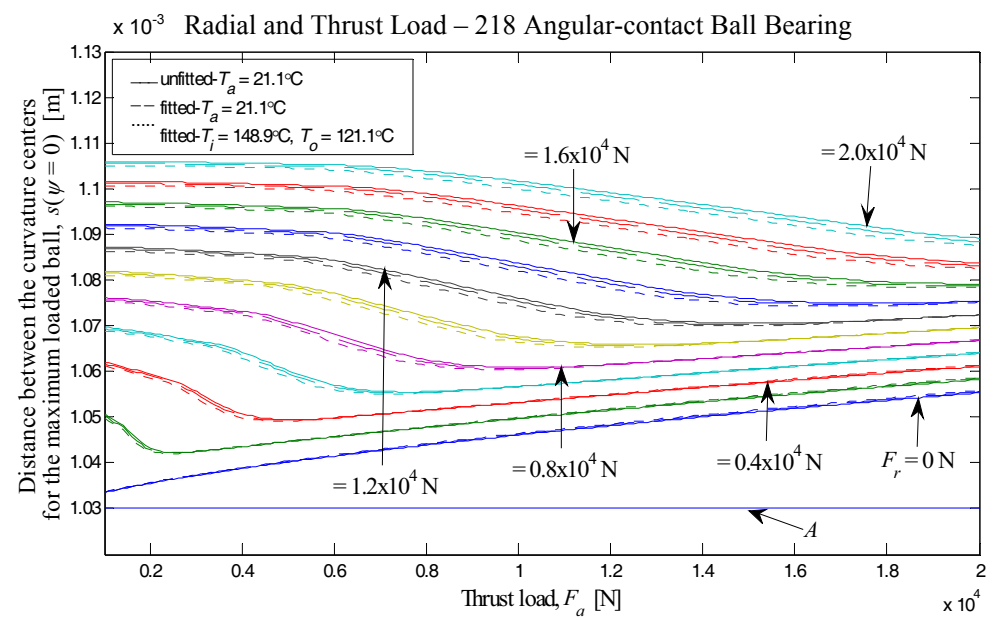

Figure 7: Distance between the curvature centers for the maximum loaded ball, $s(\psi=0)$, as a function of the thrust load, $F_{a}$. 


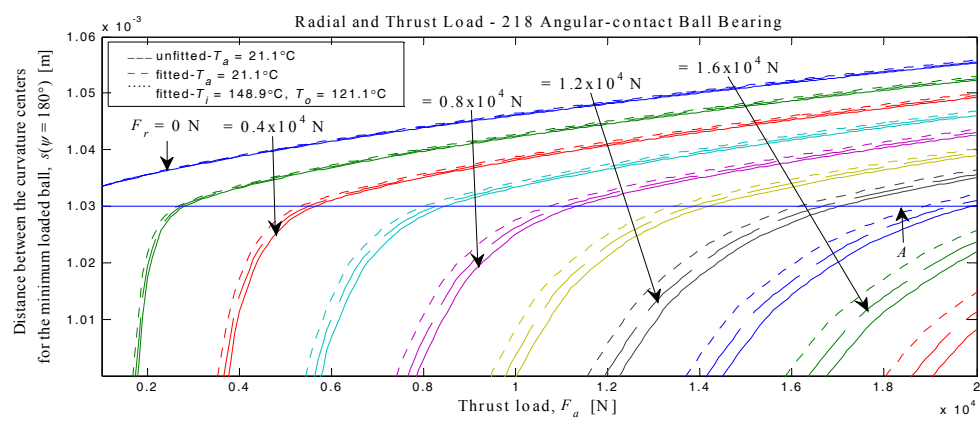

Figure 8: Distance between the curvature centers for the minimum loaded ball, $s\left(\psi=180^{\circ}\right)$, as a function of the thrust load, $F_{a}$.

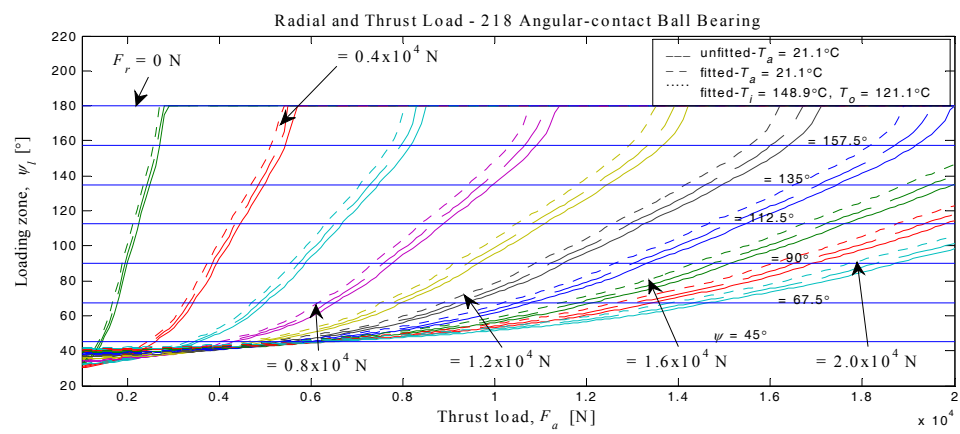

Figure 9: Loading zone, $\psi_{l}$, as a function of the thrust load, $F_{a}$.

Fig. 9 shows the loading zone. The straight lines represent the angular ball positions. Under radial load, increasing the thrust load there is an increase of the loading angle, whose maximum value is $180^{\circ}$.

Figs. 10 and 11 show the axial and radial deflections, respectively.

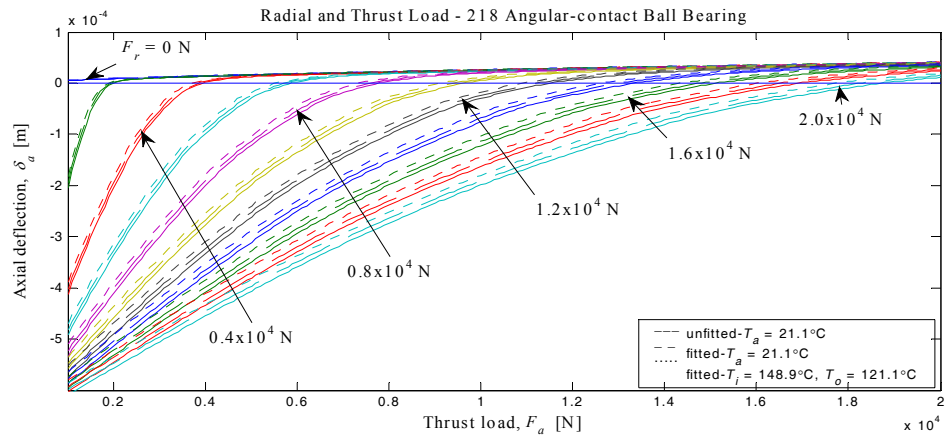

Figure 10: Axial deflection, $\delta_{a}$, as a function of the thrust load, $F_{a}$. 


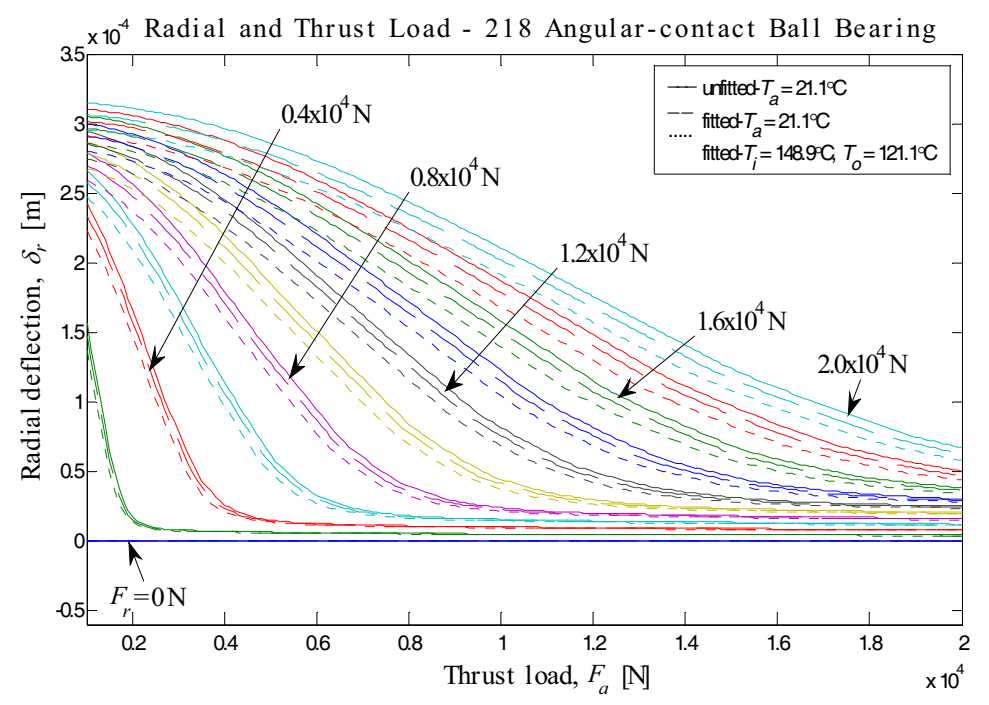

Figure 11: Radial deflection, $\delta_{r}$, as a function of the thrust load, $F_{a}$.

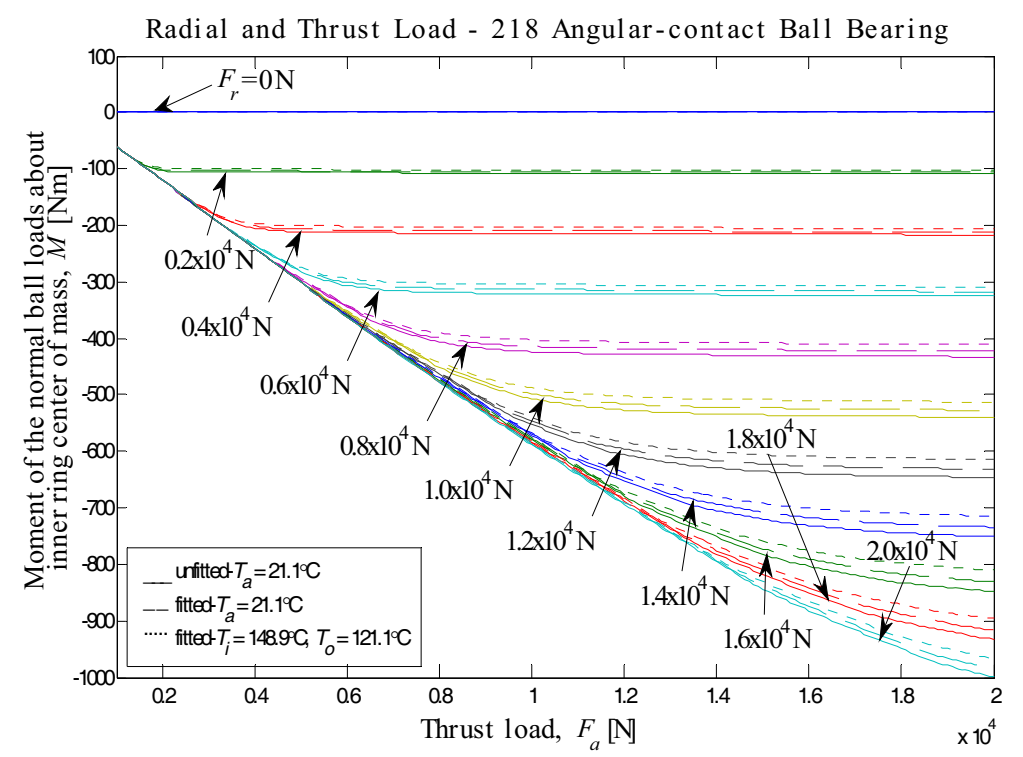

Figure 12: Moment of the normal ball loads about inner ring center of mass, $M$, as a function of the thrust load, $F_{a}$.

Fig. 12 shows the moment, $M$, such must be exerted by the thrust load, $F_{a}$, and/or the radial load, $F_{r}$, about of the inner ring center of mass, in order of prevent the angular displacement of the inner ring with respect the outer ring. 


\section{Conclusion}

This work used a procedure for get numerically, accurately and quickly, the static load distribution of a ball bearing under axial and radial loading, taking into account the influence of fits and thermal gradients. Precise applications, as for example, space applications, require a precise determination of the static loading. Models available in literature are approximate and often are not compatible with the desired degree of accuracy. This work can be extended to determine the loading on high-speed bearings where centrifugal and gyroscopic forces do not be discarded. The results of this work can be used in the accurate determination of the friction torque of the ball bearings, under any operating condition of temperature and speed.

\section{References}

[1] Stribeck, R., Ball Bearings for Various Loads. Trans. ASME 29, pp. 420463, 1907.

[2] Sjoväll, H., The Load Distribution within Ball and Roller Bearings under Given External Radial and Axial Load. Teknisk Tidskrift, Mek., h.9, pp. 97-102, 1933. (http://runeberg.org/tektid/1933m/0099 .html)

[3] Jones, A., Analysis of Stresses and Deflections, New Departure Engineering Data: Bristol, 1946.

[4] Rumbarger, J., Thrust Bearings with Eccentric Loads. Machine Design, Feb. 15, 1962.

[5] Harris, T., Rolling Bearing Analysis, $4^{\text {th }}$ ed., John Wiley \& Sons Inc.: New York, 2001.

[6] Ricci, M. C., Ball bearings subjected to a variable eccentric thrust load. DINCON'09 Proceedings of the $8^{\text {th }}$ Brazilian Conference on Dynamics, Control and Applications, May, 18-22, Bauru, Brazil, 2009. ISBN: 978-8586883-45-3.

[7] Ricci, M. C., Internal loading distribution in statically loaded ball bearings, ICCCM09 $1^{\text {st }}$ International Conference on Computational Contact Mechanics, Program and Abstracts, p. 21-22, Sept. 16-18, Lecce, Italy, 2009.

[8] Ricci, M. C., Internal loading distribution in statically loaded ball bearings subjected to a combined radial and thrust load. 6th ICCSM Proceedings of the 6th International Congress of Croatian Society of Mechanics, Sept. 30 to Oct. 2, Dubrovnik, Croatia, 2009. ISBN 978-953-7539-11-5.

[9] Ricci, M. C., Internal loading distribution in statically loaded ball bearings subjected to a combined radial, thrust, and moment load. Proceedings of the 60th International Astronautical Congress, October, 12-16, Daejeon, South Korea, 2009. ISSN 1995-6258.

[10] Ricci, M. C., Internal loading distribution in statically loaded ball bearings subjected to an eccentric thrust load. Accepted to publication at The Mathematical Problems in Engineering, 2009. 
[11] Ricci, M. C., Internal loading distribution in statically loaded ball bearings subjected to a combined radial, thrust, and moment load, including the effects of temperature and fit. Presented at 11th Pan-American Congress of Applied Mechanics, January, 04-10, Foz do Iguaçu, Brazil, 2010.

[12] Hamrock, B. J. and Anderson, W. J., Arched-Outer-Race Ball-Bearing Considering Centrifugal Forces. NASA TN D-6765, 1972.

[13] Hertz, H., On the contact of Rigid Elastic Solids and on Hardness. in Miscellaneous Papers, MacMillan, London. 163-183, 1896.

[14] Hamrock, B. J. and Anderson, W. J., Rolling-Element Bearings. NASA RP $1105,1983$. 